\title{
Acupuncture Treatment Reduced the Risk of Coronary Heart Disease in Patients with Depression: A Propensity-Score Matched Cohort Study
}

\author{
Chia-Yu Huang (D) \\ Ming-Cheng Huang \\ Mao-Feng Sun \\ Cheng-Li Lin \\ Mei-Yao Wu (DD \\ Wu-Chou Lin* \\ Hung-Rong Yen (iD)
}

'Department of Family Medicine, Taichung Tzu Chi Hospital, Buddhist Tzu Chi Medical Foundation, Taichung, 427, Taiwan; ${ }^{2}$ School of Medicine, Tzu Chi University, Hualien,

970, Taiwan; ${ }^{3}$ Graduate Institute of Chinese Medicine, School of Chinese Medicine,

College of Chinese Medicine, China Medical University, Taichung, 404, Taiwan;

${ }^{4}$ Department of Chinese Medicine, China Medical University Hospital, Taichung, 404,

Taiwan; ${ }^{5}$ Management Office for Health

Data, China Medical University Hospital,

Taichung, 404, Taiwan; ${ }^{6}$ College of

Medicine, China Medical University,

Taichung, 404, Taiwan; ${ }^{7}$ School of Post-

baccalaureate Chinese Medicine, College of

Chinese Medicine, China Medical

University, Taichung, 404, Taiwan;

${ }^{8}$ Department of Obstetrics and

Gynecology, China Medical University Hospital, Taichung, 404, Taiwan; ${ }^{9}$ Research

Center for Traditional Chinese Medicine,

Department of Medical Research, China

Medical University Hospital, Taichung, 404,

Taiwan; ${ }^{10} \mathrm{Chinese}$ Medicine Research

Center, China Medical University, Taichung, 404, Taiwan

*These authors contributed equally to this work

Correspondence: Hung-Rong Yen

Tel +886-4-22053366 ext. 33I3

Email hungrongyen@mail.cmu.edu.tw

Wu-Chou Lin

Tel +886-4-22052I 21

Email d0562@mail.cmuh.org.tw
Background: Major depressive disorder is a significant public health concern, which often leads to loss of productivity, functional decline, and various complications. The aim of this study was to investigate the effectiveness of acupuncture in the risk reduction of coronary heart disease (CHD) in patients with depression.

Methods: We enrolled patients diagnosed with depression between January 1, 1997, and December 31, 2010, through the Taiwanese National Health Insurance Research Database (NHIRD). Propensity score was used to match equal numbers $(n=14,647)$ of acupuncture cohort and no-acupuncture cohort based on characteristics including sex, age, baseline comorbidity and medication. Patients were followed up until December 31, 2013, or withdrawn from the NHIRD. The Cox regression model was used to compare the hazard ratios (HRs) of CHD in the two cohorts.

Results: The basic characteristics of the two groups were similar. A lower cumulative incidence of CHD was noted in the acupuncture cohort (Log rank test, $p<0.001)$. Over time, 1626 patients in the acupuncture cohort (21.05 per 1000 person-years) and 2412 patients in the no-acupuncture cohort (39.84 per 1000 person-years) developed CHD (adjusted HR $=0.50,95 \%$ CI $0.47-0.53$ ). The decreased CHD incidence was independent of age, sex, comorbidities, and medications used. The cumulative incidence of CHD was significantly lower in the acupuncture cohort than in the no-acupuncture cohort (Log rank test, $\mathrm{p}<0.001)$.

Conclusion: The results provided a real-world evidence that acupuncture may have beneficial effect on CHD risk reduction in patients with depression.

Keywords: acupuncture, depression, coronary heart disease, National Health Insurance Research Database, real-world evidence

\section{Introduction}

Major depression is the third cause of burden of disease worldwide and will rank first by $2030 .{ }^{1}$ Moreover, depression poses significant impact on quality of life and carries a risk for heart disease ${ }^{2}$ and stroke. ${ }^{3}$ Acupuncture is a traditional Chinese intervention for disease treatment and has been practiced for thousands of years. ${ }^{4}$ Patients with injury, musculoskeletal diseases, and neurological diseases represent the majority who receive acupuncture treatment. ${ }^{5,6}$

Patients with depression have a higher risk of coronary heart disease (CHD). ${ }^{7}$ Depression is also a debilitating comorbidity of heart failure. ${ }^{8}$ There have been a couple possible mechanisms to explain the link between depression and CHD. 
Depression is associated with the abnormal secretion of neurotransmitters, including serotonin, norepinephrine and dopamine. $^{9}$ Abnormal platelet physiology and proinflammatory factors have been suggested as potential factors leading to $\mathrm{CHD} .{ }^{10}$ Dysfunction of platelets by high concentrations of serotonin is one of the explanation. ${ }^{11}$ The population attributable risk of CHD in people with depression was reported to be $32.5 \%$, which is higher than other risk factors (eg, obesity: $20 \%$, hypertension: $17.9 \%) .{ }^{12}$ The hazard ratio (HR) of all-cause mortality of CHD was 1.16 in patients with depression. ${ }^{13}$

Selective serotonin reuptake inhibitors (SSRIs), serotonin-norepinephrine reuptake inhibitors (SNRIs), serotonin antagonists and reuptake inhibitors (SARIs), norepinephrine-dopamine reuptake inhibitors (NDRIs), tricyclic antidepressants (TCAs), noradrenergic and specific serotonergic antidepressants (NaSSAs) and monoamine oxidase inhibitors (MAOIs) are common antidepressant medications. ${ }^{14}$ TCAs have cardiac toxicity, and SNRIs have complications related to hypertension. ${ }^{15,16}$ Patients should be closely observed when they have these prescriptions. There is no evidence that shows that patients with depression have a reduced risk of CHD with the other antidepressants. Thus, clinical doctors have a great interest in finding a treatment not only for treating depression but also for reducing CHD risk. SSRIs have been recommended by the American Heart Association (AHA) for treating CHD patients with depression because of their safety. ${ }^{17}$ Although some studies have shown the effectiveness of acupuncture in patients with depression, ${ }^{18,19}$ less evidence could be found from long-term follow-up studies to show that patients with depression have CHD protection with acupuncture treatment. The National Health Insurance (NHI) program was established in 1995 in Taiwan by the National Health Insurance Administration. ${ }^{20}$ Since 1996, traditional Chinese medicine (TCM) services have been reimbursed through the NHI program, which covers more than $99 \%$ of the Taiwanese population. ${ }^{21,22}$ All of the claims data were deidentified and then stored in the Taiwanese National Health Insurance Research Database (NHIRD) for research purpose. ${ }^{20}$ The large-scale real-world data without sampling bias is one of the advantages of this database. ${ }^{23}$ In this study, we aimed to determine if patients with depression could have lower CHD risk when they received acupuncture therapy. Samples of one million people in the NHIRD were randomly selected and enrolled in the study for further investigation.

\section{Materials and Methods Data Sources}

A nationwide, population-based, 1:1 propensity scorematched cohort study by Insurance Database 2000, ${ }^{21,24,25}$ which contains all of the original claims data of 1 million beneficiaries randomly sampled from the registry of all beneficiaries in 2000, was our data source. ${ }^{22}$ There was no significant difference in our sample and the general population in age, sex, birth year, or average insured payroll-related costs. Diagnosis was coded by the International Classification of Diseases, Ninth Revision, Clinical Modification (ICD9-CM). Informed consents were waived because these were de-identified secondary data from the NHIRD. The Research Ethics Committee of China Medical University and Hospital in Taiwan approved this study (CMUH104-REC2-115).

\section{Study Cohort Identification}

We identified 55,176 patients who were newly diagnosed with depression (ICD-9-CM codes 296.2-296.3, 300.4 and 311) from 1 January 1997 to 31 December 2010 (Figure 1). Patients younger than 18 years, patients with incomplete data on age and sex during the follow-up period were excluded. Patients received acupuncture therapies from the initial diagnosis of depression through 31 December 2010 were included in the acupuncture cohort; those who did not accept acupuncture therapy during the same follow-up period were defined as no-acupuncture cohort. We included 43,395 patients, 18,912 patients in the acupuncture cohort and 24,483 patients in the no-acupuncture cohort. Random selection was performed using a 1:1 propensity score match to minimize the differences in the basic data, and the number of participants in each cohort was 14,647. Propensity score approaches were processed to minimize confounding by the indication of acupuncture therapy. One to one propensity score matching was conducted by sex, age, comorbidities, and prescription medications through multiple logistic regression analysis. ${ }^{26}$ Eventually, the numbers of participants in the acupuncture and no-acupuncture cohorts were the same. Immortal time was the period between the first acupuncture therapy and the date of initial diagnosis with depression. Patients were followed up until December 31, 2013, or withdrawn from the NHIRD.

\section{Covariate Assessment}

Age and sex are the sociodemographic factors. We divided patients into three groups by to age (18-39 years, 40-59 years, and $\geq 60$ years). Comorbidities, such as diabetes 
mellitus (DM; ICD-9-CM code 250), hypertension (ICD9-CM codes 401-405), hyperlipidemia (ICD-9-CM code 272), congestive heart failure (CHF; ICD-9-CM codes 402.01, 402.11, 402.91, 404.01, 404.03, 404.11, 404.13, 404.91, 404.93, and 428.0), anxiety (ICD-9-CM codes $300.0,300.2,300.3,308.3$, and 308.91), alcoholism or alcohol-related disorders (ICD-9-CM codes 291, 303, 305.00-305.03, 790.3, and V11.3), tobacco dependence (ICD-9-CM code 305.1), and obesity (ICD-9-CM codes 278 and A183) were taken into consideration when ICD9-CM codes appeared more than 1 time in outpatient or inpatient records before the primary diagnosis of depression. The drugs used included nonsteroidal antiinflammatory drugs (NSAIDs), oral steroids, statins, SSRIs (escitalopram, fluvoxamine, sertraline), MAOIs (selegiline, isocarboxazid,tranylcypromine, phenelzine, moclobemide), TCAs (amoxapine, desipramine, imipramine, doxepin, clomipramine, trimipramine) and other antidepressants (SNRIs: venlafaxine, duloxetine, milnacipran; NDRI: bupropion; SARI: mesyrel; NaSSA: mirtazapine), which were collected after initial diagnosis of depression. ${ }^{27}$ Among the conventional medication for depression, agomelatine is one of the FDA-approved medications for depression treatment. It is a melatonergic agonist and 5HT2c antagonist that could improve sleep patterns and the release of norepinephrine and dopamine. ${ }^{28}$ However, agomelatine was not reimbursed by the National Health Insurance program in Taiwan until 2012, therefore it was not included in our study.

\section{Types of Acupuncture and Disease Categories in the Acupuncture Cohort}

Acupuncture types were identified by the treatment codes, including manual acupuncture of TCM type (B41, B42, B45, B46, B80, B81, B82, B83, B84, B90, B91,B92, B93, B94, P27041, P31103, P32103, and P33031) and electroacupuncture (B43, B44, B86, B87, B88, B89, and P33032). We classified disease categories for patients with depression accepted acupuncture therapy by ICD9-CM codes. When patients had treatment course of acupuncture, more than one ICD-9-CM code may be found that numbers of total participants were more than acupuncture cohort only.

\section{Outcome Measurement}

The index date was defined as the first time that the patients started to receive acupuncture and the immortal time was defined as the period from the initial diagnosis of depression to the index date. The occurrence of CHD (ICD-9-CM code: ICD-9-CM: 410.9, 411.1, 413, 414.0, 414.8 and 414.9) after the index date was measured. The outcome measurement was the comparison of incidences of CHD in the two cohorts with the variable of comorbidities and drug used before the end of the study (December 31, 2013).

\section{Statistical Analyses}

Baseline characteristics of the acupuncture and noacupuncture cohorts were compared by standardized mean differences (SMD). Negligible difference in mean values or proportions between the two cohorts was defined as less than 0.1 standard deviation (SD). HR and 95\% confidence interval (CI) were calculated for each variable item by Cox proportional hazards regression. KaplanMeier method and the logrank test were conducted to find the difference between two cohorts in the development of CHD. Individuals were censored at death, loss of follow-up, withdrawal from the insurance system, or the end of 2013, whichever came first. Statistical analysis and figures were performed by SAS 9.4 (SAS Institute, Cary, NC, USA) and R software (R Foundation for Statistical Computing, Vienna, Austria). Statistical significance was defined as $\mathrm{p}<0.05$ in two-tailed tests.

\section{Results}

Random selection was performed using a 1:1 propensity score match to minimize the differences in the basic data, and the number of participants in each cohort was 14,647 (Figure 1).

Table 1 shows the baseline characteristics of both cohorts. The distributions of sex, age, comorbidities, and prescription in these two cohorts were similar. Female participants were the majority in both cohorts, and most patients were between 40 and 59 years old. Hypertension was the most common comorbidity; more than $20 \%$ of patients had this problem. In the patients with depression, $12 \%$ had DM, 19\% had hyperlipidemia, and 1\% had CHF and $1 \%$ had anxiety. There was no difference in the proportions of alcoholism or alcohol-related disorders, tobacco dependence, and obesity in the two cohorts. NSAIDs were most common medications in both cohorts, and almost all patients had this prescription. Of the participants in these cohorts, $76 \%$ used oral steroids, $17 \%$ had statin agents, $62 \%$ were treated by SSRIs, $8 \%$ had MAOIs prescriptions and 34\% received TCAs treatment. Manual 


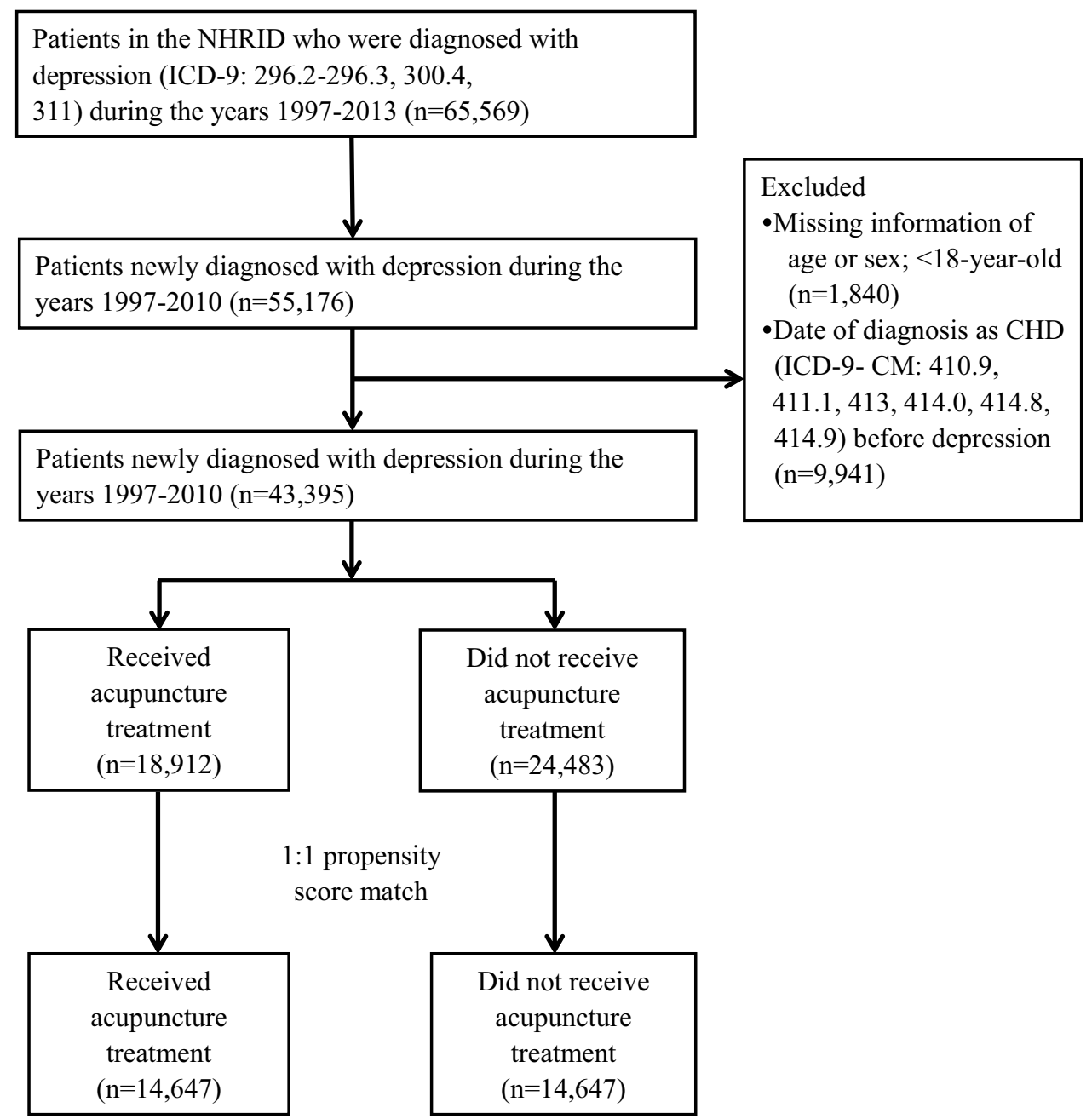

Figure I A total of 43,395 patients with depression were newly diagnosed from 1997 to 2010 . Sex, age, comorbidities, and prescription drugs used were processed via propensity-score matching I:I by 14,647 patients who were included in both the acupuncture cohort and the no-acupuncture cohort.

Abbreviation: LHID 2000, Longitudinal Health Insurance Database 2000.

acupuncture was the most common manipulation, and $88 \%$ of the patients had this experience.

Electroacupuncture was performed in 3\% of the participants. Another $9 \%$ of patients were treated by combination of acupuncture with their prescriptions. The mean duration between when the patients had been diagnosed with depression and the first time receiving acupuncture therapy was approximately 1320 days. The mean number of acupuncture visits was 8.71 .

A total of 4038 patients developed CHD events during the follow-up period (Table 2). Patients with depression had a lower risk of developing CHD after they received acupuncture treatment, and the adjusted HR was 0.47 (95\% CI 0.44-0.51). Figure 2 shows that the cumulative incidence of CHD was significantly lower in the acupuncture cohort (log-rank test, $\mathrm{p}<0.001$ ).
Table 3 shows the 1626 patients in the acupuncture cohort (21.05 per 1000 person-years) and 2412 patients in the no-acupuncture cohort (39.84 per 1000 personyears) who developed CHD (adjusted HR 0.50, 95\% CI 0.47-0.53). The benefit for lowering CHD incidence was observed in male and female groups: the adjusted HR was 0.47 in females, 95\% CI $0.43-0.51$; adjusted HR was 0.56 in males, $95 \%$ CI $0.50-0.62$. The effectiveness of acupuncture therapy could also be found in different age groups (adjusted HR 0.53, 95\% CI $0.45-$ 0.62 in the 18-39-year-old group; adjusted HR 0.48 , 95\% CI $0.44-0.52$ in the 40-59-year-old group; adjusted HR $0.54,95 \%$ CI $0.47-0.62$ in the over 60 -year-old group). Whether they were patients with comorbidities or not, acupuncture therapy was helpful for decreasing the risk of CHD. Coprescription with steroids, NSAIDs 
Table I Characteristics of Patients with Depression Who Received Acupuncture Therapy and Patients with Depression Who Did Not Receive Acupuncture Therapy

\begin{tabular}{|c|c|c|c|c|c|}
\hline \multirow[t]{3}{*}{ Variable } & \multicolumn{4}{|c|}{ Accepted Acupuncture } & \multirow[t]{3}{*}{ Standardized Mean Difference } \\
\hline & \multicolumn{2}{|c|}{$\begin{array}{c}\text { No } \\
(n=\mid 4,647)\end{array}$} & \multicolumn{2}{|c|}{$\begin{array}{c}\text { Yes } \\
(n=\mid 4,647)\end{array}$} & \\
\hline & $\mathbf{n}$ & $\%$ & $\mathbf{n}$ & $\%$ & \\
\hline Gender & & & & & 0.4746 \\
\hline Women & 9295 & 63.46 & 9236 & 63.06 & \\
\hline Men & 5352 & 36.54 & 5411 & 36.94 & \\
\hline Age group & & & & & 0.99 \\
\hline $18-39$ & 6630 & 45.27 & 6630 & 45.27 & \\
\hline $40-59$ & 6652 & 45.42 & 6652 & 45.42 & \\
\hline$\geq 60$ & 1365 & 9.32 & 1365 & 9.32 & \\
\hline Mean $\pm S D$ (years) & \multicolumn{2}{|c|}{$43.19(14.86)$} & \multicolumn{2}{|c|}{$43.20(|4.8|)$} & 0.9427 \\
\hline \multicolumn{6}{|l|}{ Baseline Comorbidity } \\
\hline Diabetes mellitus & 1746 & 11.92 & 1779 & 12.15 & 0.5534 \\
\hline Hypertension & 3262 & 22.27 & 3384 & 23.1 & 0.0888 \\
\hline Hyperlipidemia & 2783 & 19 & 2752 & 18.79 & 0.6436 \\
\hline Congestive heart failure & 169 & 1.15 & 175 & 1.19 & 0.7449 \\
\hline Anxiety & 107 & 0.73 & 101 & 0.69 & 0.6763 \\
\hline Alcoholism & 274 & 1.87 & 261 & 1.78 & 0.5705 \\
\hline Tobacco used & 104 & 0.71 & 104 & 0.71 & 0.99 \\
\hline Obesity & 130 & 0.89 & 125 & 0.85 & 0.7532 \\
\hline \multicolumn{6}{|l|}{ Drug used } \\
\hline Oral steroids & 11,108 & 75.84 & $|I| 3 \mid$, & 76 & 0.7533 \\
\hline NSAIDs & 14,516 & 99.11 & 14,516 & 99.11 & 0.99 \\
\hline Statins & 2534 & 17.3 & 2534 & 17.3 & 0.99 \\
\hline SSRIs & 9095 & 62.09 & 9035 & 61.68 & 0.4704 \\
\hline MAOls & 1179 & 8.05 & 1162 & 7.93 & $0.7|4|$ \\
\hline TCAs & 4999 & 34.13 & 4999 & 34.13 & 0.99 \\
\hline Other antidepressant drugs & 4757 & 32.48 & 4653 & 31.77 & 0.1932 \\
\hline \multicolumn{6}{|l|}{ Types of acupuncture } \\
\hline Manual acupuncture & - & - & 12,842 & 87.68 & \\
\hline Electroacupuncture & - & - & 418 & 2.85 & \\
\hline Combination of manual acupuncture and electroacupuncture & - & - & 1387 & 9.47 & \\
\hline Duration between depression date and index, days (mean, median) & \multicolumn{2}{|c|}{$1315.98(1031)$} & \multicolumn{2}{|c|}{$1322.59(979)$} & \\
\hline Acupuncture visits, (mean, median) & & & \multicolumn{2}{|c|}{$8.71(3)$} & \\
\hline
\end{tabular}

Note: The mean (median) of follow-up period were 4.13 (3.35) and 5.27 (4.59) years for acupuncture cohort and compared cohort.

Abbreviations: SD, standard deviation; NSAIDs, nonsteroidal anti-inflammatory drugs; SSRIs, selective serotonin reuptake inhibitors; MAOls, monoamine oxidase inhibitors; TCAs, tricyclic antidepressants.

or statins did not change the positive results of acupuncture therapy. With the help of acupuncture treatment, lower CHD risk could still be found in patients prescribed different types of antidepressant medications. Table 4 also reveals depression patients had lower incidence of developing CHD (estimate subhazard ratio
$0.52,95 \%$ CI $0.49-0.56)$ through competing-risks regression models. ${ }^{29}$

\section{Discussion}

This is the first study to demonstrate that the CHD risk in depression patients could be improved by acupuncture 
Table 2 Cox Model with Hazard Ratios and 95\% Confidence Intervals of Coronary Heart Disease Associated with Receiving Acupuncture and Covariates Among Patients with Depression

\begin{tabular}{|c|c|c|c|c|}
\hline Variable & $\begin{array}{l}\text { No. of Event } \\
\quad(n=4038)\end{array}$ & HR & $(95 \% \mathrm{Cl})$ & p-value \\
\hline \multirow{2}{*}{\multicolumn{5}{|c|}{$\begin{array}{l}\text { Accepted } \\
\text { Acupuncture }\end{array}$}} \\
\hline & & & & \\
\hline No & 2412 & 1.00 & reference & \\
\hline Yes & 1626 & 0.47 & $(0.44-0.5 \mathrm{I})$ & $<0.0001$ \\
\hline
\end{tabular}

Note: HR represented relative hazard ratio.

Abbreviations: $\mathrm{HR}$, hazard ratio; $\mathrm{Cl}$, confidence interval.

treatment. Mental disorders, which included depression, is one of the common disease categories among acupuncture visits in Taiwan. ${ }^{6}$ The risk of CHD has never been mentioned as a result of acupuncture therapy in patients with depression. According to our results, more than half of the patients with depression have received acupuncture therapy in Taiwan. With the analysis of this real-world data with long-term follow-up, we were able to show that the benefit of acupuncture intervention was independent of sex, age, comorbidities and drugs use (oral steroids, NSAIDs, statins and SSRIs).

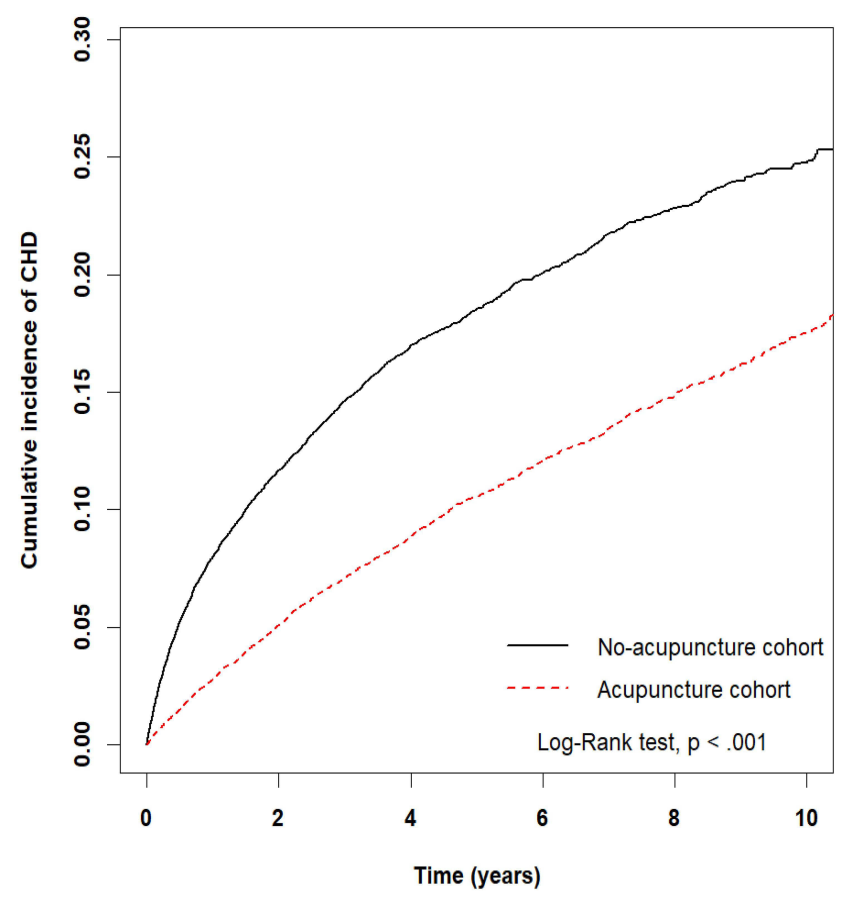

Figure 2 Cumulative incidence of coronary heart disease in acupuncture (dashed line) and the no-acupuncture cohort (solid line). Patients in acupuncture group had lower incidence of CHD significantly (Log rank test, $\mathrm{p}<0.00 \mathrm{I}$ ).

Abbreviation: CHD, coronary heart disease.
A couple mechanisms might explain why acupuncture exerts protective effect in reducing the risk of CHD in patients with depression. An earlier study reported that acupuncture have advantages in left ventricular function in patients with coronary artery disease. ${ }^{30}$ Recently, acupuncture was also demonstrated to be effective and safe in reducing frequency of angina attacks in patients with chronic stable angina. ${ }^{31}$ Symptoms of depression are associated with several neurotransmitters, such as serotonin, norepinephrine and dopamine. ${ }^{9}$ Serotonin is a vasoactive substance that has been identified in the brain, colon, and platelets. ${ }^{32}$ In the cardiac system, it has also been noted in the vascular beds and sympathetic nerve. Serotonin is important in the pathophysiology of atherosclerosis because it can promote platelet aggregation and arterial vasoconstriction. $^{33-35}$ Stimulation via acupuncture might be able to regulate the serum level of serotonin. ${ }^{36}$ Another hypothesis is that dysfunction of the hypothalamicpituitary-adrenal (HPA) axis contributes to depression. Stimulating hypothalamic and hippocampal regions by acupuncture has been shown to be helpful for stabilizing the HPA axis and normalizing the secretion of neurotransmitters. ${ }^{37}$

Vascular endothelial growth factor (VEGF) is a cellular mitogen. Its role in hippocampal neurogenesis is believed to be a response to stress. Some studies have found a relationship between VEGF and depression, and suggest that VEGF may be a new target for the treatment of depression. ${ }^{38}$ Endothelial progenitor cells (EPCs) have beneficial effects in atherosclerosis, angiogenesis, and vascular repair. The effects of acupuncture on EPCs in patients with coronary heart disease through the VEGF pathway has been reported. ${ }^{39}$ The regulation of VEGF may be a possible mechanism to prevent CHD in depression patients with acupuncture therapy, and further evaluations are warranted to provide more rigorous evidence.

Sleep problems, such as sleep apnea and short sleep duration, could contribute to CHD. ${ }^{40,41}$ Improvement of sleep disturbance is beneficial for the management of depression, as demonstrated by the effectiveness of agomelatine. $^{28}$ Treatment of sleep disorders is also an indication of acupuncture therapy, ${ }^{42,43}$ and this implies that acupuncture therapy may have indirect effects on the prevention of CHD in patients with depression.

Not only CHD but also comorbidities of depression, including DM, hypertension and CHF, have relationships with inflammation. ${ }^{4,45}$ Acupuncture has been found to not only exert anti-nociceptive effect but also suppress 
Table 3 Incidence Rates, Hazard Ratios and Confidence Intervals of Coronary Heart Disease for Patients with Depression Who Received and Did Not Receive Acupuncture, Stratified by Sex, Age, Comorbidities and Prescription Drugs Used

\begin{tabular}{|c|c|c|c|c|c|c|c|c|}
\hline \multirow[t]{4}{*}{ Variables } & \multicolumn{6}{|c|}{ Accepted Acupuncture } & \multicolumn{2}{|c|}{$\begin{array}{l}\text { Compared with No-Acupuncture } \\
\text { Users }\end{array}$} \\
\hline & \multicolumn{3}{|c|}{ No } & \multicolumn{3}{|c|}{ Yes } & \multirow[t]{2}{*}{ Crude HR } & \multirow[t]{2}{*}{ Adjusted $\mathbf{H R}^{\dagger}$} \\
\hline & \multicolumn{3}{|c|}{$(n=14,647)$} & \multicolumn{3}{|c|}{$(n=14,647)$} & & \\
\hline & Event & Person Years & IR & Event & Person Years & IR & $(95 \% \mathrm{Cl})$ & $(95 \% \mathrm{Cl})$ \\
\hline Total & 2412 & 60,539 & 39.84 & 1626 & 77,231 & 21.05 & $0.56(0.53-0.60)^{* * *}$ & $0.50(0.47-0.53)^{* * *}$ \\
\hline \multicolumn{9}{|l|}{ Gender } \\
\hline Women & 1569 & 37,945 & 41.35 & 987 & 48,220 & 20.47 & $0.52(0.48-0.57)^{* * *}$ & $0.47(0.43-0.5 \mathrm{I})^{* * *}$ \\
\hline Men & 843 & 22,594 & 37.31 & 639 & 29,010 & 22.03 & $0.62(0.56-0.69)^{* * *}$ & $0.56(0.5-0.62)^{* * * *}$ \\
\hline \multicolumn{9}{|l|}{ Age group } \\
\hline $18-39$ & 356 & 29,622 & 12.02 & 239 & 35,310 & 6.77 & $0.58(0.49-0.68)^{* * *}$ & $0.53(0.45-0.62)^{* * *}$ \\
\hline $40-59$ & 1615 & 26,805 & 60.25 & 1038 & 35,337 & 29.37 & $0.52(0.48-0.56)^{* * *}$ & $0.48(0.44-0.52)^{* * *}$ \\
\hline$\geq 60$ & $44 I$ & 4112 & 107.24 & 349 & 6584 & 53.01 & $0.56(0.49-0.65)^{* * *}$ & $0.54(0.47-0.62)^{* * * *}$ \\
\hline \multicolumn{9}{|c|}{ Baseline Comorbidity } \\
\hline \multicolumn{9}{|c|}{ Diabetes mellitus } \\
\hline No & 1884 & 54,457 & 34.60 & 1225 & 68,358 & 17.92 & $0.54(0.5 \mathrm{I}-0.59)^{* * *}$ & $0.49(0.45-0.52)^{* * *}$ \\
\hline Yes & 528 & 6082 & 86.81 & 401 & 8873 & 45.20 & $0.57(0.5-0.65)^{* * *}$ & $0.54(0.47-0.6 \mathrm{I})^{* * * *}$ \\
\hline \multicolumn{9}{|l|}{ Hypertension } \\
\hline No & 1305 & 49,451 & 26.39 & 831 & 60,307 & 13.78 & $0.54(0.5-0.59)^{* * *}$ & $0.5(0.46-0.55)^{* * * *}$ \\
\hline Yes & 1107 & 11,088 & 99.84 & 795 & 16,924 & 46.98 & $0.52(0.47-0.57)^{* * *}$ & $0.5(0.45-0.55)^{* * *}$ \\
\hline \multicolumn{9}{|l|}{ Hyperlipidemia } \\
\hline No & 1552 & 50,827 & 30.53 & 1043 & 64,033 & 16.29 & $0.56(0.52-0.61)^{* * *}$ & $0.5(0.46-0.54)^{* * *}$ \\
\hline Yes & 860 & 9712 & 88.55 & 583 & 13,198 & 44.17 & $0.54(0.48-0.6)^{* * *}$ & $0.48(0.44-0.54)^{* * *}$ \\
\hline \multicolumn{9}{|c|}{ Congestive heart failure } \\
\hline No & 2341 & 60,116 & 38.94 & 1583 & 76,471 & 20.70 & $0.56(0.53-0.6)^{* * *}$ & $0.5(0.47-0.54)^{* * *}$ \\
\hline Yes & 71 & 423 & 167.70 & 43 & 759 & 56.62 & $0.38(0.26-0.56)^{* * *}$ & $0.44(0.29-0.65)^{* * *}$ \\
\hline \multicolumn{9}{|l|}{ Anxiety } \\
\hline No & 2391 & 60,145 & 39.75 & 1609 & 76,745 & 20.97 & $0.56(0.52-0.59)^{* * *}$ & $0.50(0.47-0.53)^{* * *}$ \\
\hline Yes & 21 & 394 & 53.28 & 17 & 486 & 34.97 & $0.73(0.39-1.4)$ & $0.69(0.34-1.37)$ \\
\hline \multicolumn{9}{|l|}{ Alcoholism } \\
\hline No & 2383 & 59,525 & 40.03 & 1609 & $75,94 I$ & 21.19 & $0.56(0.53-0.60)^{* * *}$ & $0.50(0.47-0.54)^{* * * *}$ \\
\hline Yes & 29 & 1014 & 28.59 & 17 & 1290 & 13.18 & $0.48(0.26-0.87)^{*}$ & $0.37(0.20-0.69)^{* *}$ \\
\hline \multicolumn{9}{|l|}{ Tobacco used } \\
\hline No & 2403 & 60,253 & 39.88 & 1622 & 76,918 & 21.09 & $0.56(0.53-0.60)^{* * *}$ & $0.50(0.47-0.53)^{* * *}$ \\
\hline Yes & 9 & 286 & 31.46 & 4 & 312 & 12.80 & $0.41(0.13-1.32)$ & $0.22(0.05-0.90)^{*}$ \\
\hline \multicolumn{9}{|l|}{ Obesity } \\
\hline No & 2390 & 60,134 & 39.74 & 1614 & 76,604 & 21.07 & $0.56(0.53-0.60)^{* * *}$ & $0.50(0.47-0.54)^{* * *}$ \\
\hline Yes & 22 & 405 & 54.29 & 12 & 627 & 19.14 & $0.39(0.19-0.79)^{* *}$ & $0.19(0.08-0.46)^{* * *}$ \\
\hline \multicolumn{9}{|l|}{ Drug used } \\
\hline \multicolumn{9}{|l|}{ Oral steroids } \\
\hline No & 792 & $|2| 6 \mid$, & 65.13 & 457 & 15,765 & 28.99 & $0.49(0.43-0.55)^{* * *}$ & $0.43(0.39-0.49)^{* * * *}$ \\
\hline Yes & 1620 & 48,378 & 33.49 & 1169 & 61,466 & 19.02 & $0.59(0.55-0.64)^{* * *}$ & $0.54(0.50-0.58)^{* * * *}$ \\
\hline
\end{tabular}


Table 3 (Continued).

\begin{tabular}{|c|c|c|c|c|c|c|c|c|}
\hline \multirow[t]{4}{*}{ Variables } & \multicolumn{6}{|c|}{ Accepted Acupuncture } & \multicolumn{2}{|c|}{$\begin{array}{l}\text { Compared with No-Acupuncture } \\
\text { Users }\end{array}$} \\
\hline & \multicolumn{3}{|c|}{ No } & \multicolumn{3}{|c|}{ Yes } & \multirow{3}{*}{$\begin{array}{l}\text { Crude HR } \\
(95 \% \mathrm{Cl})\end{array}$} & \multirow{3}{*}{$\begin{array}{c}\text { Adjusted } \mathbf{H R}^{\dagger} \\
(95 \% \mathrm{Cl})\end{array}$} \\
\hline & \multicolumn{3}{|c|}{$(n=14,647)$} & \multicolumn{3}{|c|}{$(n=14,647)$} & & \\
\hline & Event & Person Years & IR & Event & Person Years & IR & & \\
\hline \multicolumn{9}{|l|}{ NSAIDs } \\
\hline No & 54 & 241 & 223.91 & 38 & 399 & 95.32 & $0.52(0.34-0.78)^{* *}$ & $0.33(0.2 \mathrm{I}-0.53)^{* * *}$ \\
\hline Yes & 2358 & 60,298 & 39.11 & 1588 & 76,832 & 20.67 & $0.56(0.52-0.59)^{* * *}$ & $0.50(0.47-0.54)^{* * * *}$ \\
\hline \multicolumn{9}{|l|}{ Statins } \\
\hline No & $197 \mid$ & 48,983 & 40.24 & 1269 & 62,868 & 20.19 & $0.53(0.50-0.57)^{* * *}$ & $0.47(0.44-0.5)^{* * *}$ \\
\hline Yes & $44 I$ & $\mathrm{II}, 555$ & 38.16 & 357 & 14,363 & 24.86 & $0.67(0.58-0.77)^{* * *}$ & $0.64(0.55-0.73)^{* * *}$ \\
\hline \multicolumn{9}{|l|}{ SSRIs } \\
\hline No & 1406 & 21,736 & 64.69 & 842 & 29,929 & 28.13 & $0.47(0.43-0.5 \mathrm{I})^{* * *}$ & $0.44(0.40-0.48)^{* * *}$ \\
\hline Yes & 1006 & 38,803 & 25.93 & 784 & 47,302 & 16.57 & $0.66(0.60-0.73)^{* * *}$ & $0.58(0.53-0.64)^{* * *}$ \\
\hline \multicolumn{9}{|l|}{ MAOls } \\
\hline No & 2163 & 54,822 & 39.45 & $|45|$ & 70,156 & 20.68 & $0.56(0.52-0.59)^{* * *}$ & $0.50(0.47-0.53)^{* * *}$ \\
\hline Yes & 249 & 5717 & 43.56 & 175 & 7075 & 24.73 & $0.59(0.49-0.72)^{* * *}$ & $0.53(0.44-0.65)^{* * *}$ \\
\hline \multicolumn{9}{|l|}{ TCAs } \\
\hline No & 1505 & 38,617 & 38.97 & 962 & 48,656 & 19.77 & $0.54(0.50-0.59)^{* * *}$ & $0.47(0.44-0.5 \mathrm{I})^{* * *}$ \\
\hline Yes & 907 & 21,921 & 41.37 & 664 & 28,574 & 23.24 & $0.59(0.53-0.65)^{* * *}$ & $0.55(0.49-0.60)^{* * *}$ \\
\hline \multicolumn{9}{|l|}{ Other antidepressant drugs } \\
\hline No & 2092 & 40,405 & 51.78 & 1379 & 53,621 & 25.72 & $0.53(0.50-0.57)^{* * *}$ & $0.49(0.45-0.52)^{* * *}$ \\
\hline Yes & 320 & 20,134 & 15.89 & 247 & 23,610 & 10.46 & $0.67(0.57-0.79)^{* * *}$ & $0.61(0.52-0.72)^{* * *}$ \\
\hline
\end{tabular}

Notes: Adjusted $\mathrm{HR}^{\dagger}$ : adjusted for accepted acupuncture, age, gender, diabetes mellitus, hypertension, hyperlipidemia, congestive heart failure, anxiety, alcoholism, tobacco used, obesity, oral steroids, NSAIDs, statins, SSRIs, MAOIs, TCAs and other antidepressant drugs in Cox proportional hazards regression. $* \mathrm{p}<0.05$; $* * \mathrm{p}<0.01$; $* * * \mathrm{p}<0.00 \mathrm{I}$. Abbreviations: IR, incidence rate (per I,000 person-years); HR, hazard ratio; Cl, confidence interval; NSAIDs, nonsteroidal anti-inflammatory drugs; SSRIs, selective serotonin reuptake inhibitors; MAOls, monoamine oxidase inhibitors; TCAs, tricyclic antidepressants.

Table 4 Accepted Acupuncture Cohort to No-Acupuncture Cohort Subhazard Ratio of Coronary Heart Disease Estimated Using the Competing-Risks Regression Models

\begin{tabular}{|l|c|c|}
\hline \multirow{2}{*}{} & \multicolumn{2}{|c|}{ Competing-Risks Regression Models } \\
\cline { 2 - 3 } & \multicolumn{2}{|c|}{ Accepted Acupuncture } \\
\cline { 2 - 3 } & No & Yes \\
\hline Coronary heart disease & & \\
Crude SHR $(95 \% \mathrm{Cl})$ & I (Reference) & $0.57(0.54,0.6 \mathrm{I})^{* * * *}$ \\
Adjusted SHR ${ }^{\dagger}(95 \% \mathrm{Cl})$ & I (Reference) & $0.52(0.49,0.56)^{* * *}$ \\
\hline
\end{tabular}

Notes: Crude SHR, relative subhazard ratio; Adjusted $\mathrm{SHR}^{\dagger}$ : multivariable analysis included age, gender, diabetes mellitus, hypertension, hyperlipidemia, congestive heart failure, anxiety, alcoholism, tobacco used, obesity, oral steroids, NSAIDs, statins, SSRIs, MAOls, TCAs and other antidepressant drugs. $* * * \mathrm{p}<0.00 \mathrm{I}$. Abbreviations: SHR, subhazard ratio; $\mathrm{Cl}$, confidence interval.

inflammation. ${ }^{46-48}$ Through the release of calcitonin generelated peptide, vasodilatory and anti-inflammatory effects were hypothesized to be induced by acupuncture. ${ }^{49}$ Furthermore, cardiac work capacity could be improved by acupuncture in patients with angina pectoris. ${ }^{50}$ Researchers also found that acupuncture pretreatment could inhibit the $\beta 1$-adrenoceptor signaling pathway, an effect which protects the heart from myocardial ischemia injury in animal studies. ${ }^{51}$ The potential of acupuncture to protect against CHD can be found in these studies, although further investigations are needed to determine whether these mechanisms are suitable for humans.

Our study has several limitations. The claim data from NHIRD could not be validated and the severity of depression, such as suicidal ideations and social and work disability were not included. Thus, we performed a 1:1 propensity score match, which was useful to minimize the difference between two cohorts. The percentages of patients prescribed antidepressants were similar. Personal habits and some 
characteristics, such as height, weight, exercise status, smoking and drinking consumption, are difficult to identify from the NHIRD. Thus, we tried to define a diagnosis of alcoholism or alcohol-related disorders, tobacco dependence, and obesity to represent these parameters then via a 1:1 propensity score match to eliminate the difference. The distribution of patients with these problems was similar, and these comorbidities did not change the significant protective effect of acupuncture on CHD in patients with depression. The NHIRD database did not reveal the acupoints for depression treatment. And the dose of acupuncture therapy, such as frequency or duration is variable and depends on the diagnosis of the patients and the experience of the TCM doctors. Based on uniform TCM program training in Chinese medical colleges in Taiwan, most TCM doctors have basic ideas for treatment goals. But, different complaints, comorbidities and acceptance of patients could contribute to variable prescriptions of acupuncture. A retrospective study through realworld data could not offer the causal relation between acupuncture intervention and outcomes or collect adequate number of participants with same treatment course for analysis. ${ }^{52}$

\section{Conclusions}

The results of this study on CHD risk reduction demonstrate the effects and suggest advantages of acupuncture treatment in patients with depression in Taiwan. It provides noteworthy ideas for future studies to investigate the effectiveness of acupuncture to integrate to the clinical management of patients with depression.

\section{Data Sharing Statement}

The datasets we used in this study were released by the Taiwan NHIRD (http://nhird.hri.org.tw/en/indx.html), maintained and managed by National Health Research Institutes (http://www.nhri.org.tw/), Taiwan. The datasets are limited to be used for research purposes only. Applicants must follow the Computer-Processed Personal Data Protection Law (http://www.winklerpartners.com/?p= 987) and related regulations of National Health Insurance Administration and National Health Research Institutes, and the agreement must be signed by the applicant and his/her supervisor upon application submission. All applications are reviewed for approval of data release.

\section{Author Contributions}

All authors made a significant contribution to the work reported, whether that is in the conception, study design, execution, acquisition of data, analysis and interpretation, or in all these areas; took part in drafting, revising or critically reviewing the article; gave final approval of the version to be published; have agreed on the journal to which the article has been submitted; and agree to be accountable for all aspects of the work.

\section{Funding}

This work was financially supported by the "Chinese Medicine Research Center, China Medical University" from the Featured Areas Research Center Program within the framework of the Higher Education Sprout Project by the Ministry of Education (MOE) in Taiwan (CMRC-CHM-1). This study was also supported in part by China Medical University (CMU103BC-4-2), China Medical University Hospital (DMR-108-004, DMR-108-115 and DMR-108-116), Ministry of Science and Technology (108-2638-B-039-001-MY2), and health and welfare surcharge of tobacco products, China Medical University Hospital Cancer Research Center of Excellence, Ministry of Health and Welfare (MOHW110-TDU-B-212-144024), Taiwan. None of the funders and institutions listed had a role in the design and conduct of the study; collection, management, analysis, and interpretation of the data; preparation, review, or approval of the manuscript; and decision to submit the manuscript for publication.

\section{Disclosure}

The authors declare that the research was conducted in the absence of any commercial or financial relationships that could be construed as a potential conflict of interest.

\section{References}

1. Malhi GS, Mann JJ. Depression. Lancet. 2018;392(10161):2299-2312. doi:10.1016/S0140-6736(18)31948-2.

2. Sher Y, Lolak S, Maldonado JR. The impact of depression in heart disease. Curr Psychiatry Rep. 2010;12(3):255-264. doi:10.1007/ s11920-010-0116-8

3. Pan A, Sun Q, Okereke OI, Rexrode KM, Hu FB. Depression and risk of stroke morbidity and mortality: a meta-analysis and systematic review. JAMA. 2011;306(11):1241-1249. doi:10.1001/jama.2011.1282

4. Kaptchuk TJ. Acupuncture: theory, efficacy, and practice. Ann Intern Med. 2002;136(5):374-383. doi:10.7326/0003-4819-136-5-200203050-00010

5. Ernst E. Acupuncture-a critical analysis. J Intern Med. 2006;259 (2):125-137. doi:10.1111/j.1365-2796.2005.01584.x

6. Wu MY, Lee YC, Lin CL, Huang MC, Sun MF, Yen HR. Trends in use of acupuncture among adults in Taiwan from 2002 to 2011: a nationwide population-based study. PLoS One. 2018;13(4): e0195490. doi:10.1371/journal.pone. 0195490

7. Anda R, Williamson D, Jones D, et al. Depressed affect, hopelessness, and the risk of ischemic heart disease in a cohort of U.S. adults. Epidemiology. 1993;4(4):285-294. doi:10.1097/00001648-199307000-00003

8. Sbolli M, Fiuzat M, Cani D, O'Connor CM. Depression and heart failure: the lonely comorbidity. Eur J Heart Fail. 2020;22 (11):2007-2017. doi:10.1002/ejhf.1865 
9. Fava M. The role of the serotonergic and noradrenergic neurotransmitter systems in the treatment of psychological and physical symptoms of depression. J Clin Psychiatry. 2003;64Suppl 13:26-29.

10. Williams MS, Rogers HL, Wang NY, Ziegelstein RC. Do platelet-derived microparticles play a role in depression, inflammation, and acute coronary syndrome? Psychosomatics. 2014;55 (3):252-260. doi:10.1016/j.psym.2013.09.004

11. Bruce EC, Musselman DL. Depression, alterations in platelet function, and ischemic heart disease. Psychosom Med. 2005;67(Suppl 1): S34-36. doi:10.1097/01.psy.0000164227.63647.d9

12. Yusuf S, Hawken S, Ounpuu S, et al. Effect of potentially modifiable risk factors associated with myocardial infarction in 52 countries (the INTERHEART study): case-control study. Lancet. 2004;364 (9438):937-952. doi:10.1016/S0140-6736(04)17018-9

13. Ahto M, Isoaho R, Puolijoki H, et al. Stronger symptoms of depression predict high coronary heart disease mortality in older men and women. Int J Geriatr Psychiatry. 2007;22(8):757-763. doi:10.1002/gps. 1735.

14. Prado CE, Watt S, Crowe SF. A meta-analysis of the effects of antidepressants on cognitive functioning in depressed and non-depressed samples. Neuropsychol Rev. 2018;28(1):32-72. doi:10.1007/s11065-018-9369-5.

15. Glassman AH, O'Connor CM, Califf RM, et al. Sertraline treatment of major depression in patients with acute MI or unstable angina. JAMA. 2002;288(6):701-709. doi:10.1001/jama.288.6.701

16. Glassman AH, Roose SP, Bigger JT Jr. The safety of tricyclic antidepressants in cardiac patients. Risk-benefit reconsidered. JAMA. 1993;269(20):2673-2675. doi:10.1001/jama.1993.03500200087039

17. Lichtman JH, Bigger JT Jr, Blumenthal JA, et al. Depression and coronary heart disease: recommendations for screening, referral, and treatment: a science advisory from the American Heart Association Prevention Committee of the Council on Cardiovascular Nursing, Council on Clinical Cardiology, Council on Epidemiology and Prevention, and Interdisciplinary Council on Quality of Care and Outcomes Research: endorsed by the American Psychiatric Association. Circulation. 2008;118(17):1768-1775. doi:10.1161/ CIRCULATIONAHA.108.190769

18. Qu SS, Huang Y, Zhang ZJ, et al. A 6-week randomized controlled trial with 4-week follow-up of acupuncture combined with paroxetine in patients with major depressive disorder. J Psychiatr Res. 2013;47 (6):726-732. doi:10.1016/j.jpsychires.2013.02.004

19. Man SC, Hung $\mathrm{BH}, \mathrm{Ng} \mathrm{RM}$, et al. A pilot controlled trial of a combination of dense cranial electroacupuncture stimulation and body acupuncture for post-stroke depression. BMC Complement Altern Med. 2014;14(1):255. doi:10.1186/1472-6882-14-255

20. Lin LY, Warren-Gash C, Smeeth L, Chen PC. Data resource profile: the National Health Insurance Research Database (NHIRD). Epidemiol Health. 2018;40:e2018062. doi:10.4178/epih.e2018062

21. Huang CY, Wu MY, Kuo YH, Tou SI, Yen HR. Chinese herbal medicine is helpful for survival improvement in patients with multiple myeloma in Taiwan: a nationwide retrospective matched-cohort Study. Integr Cancer Ther. 2020;19:1534735420943280. doi:10.11 77/1534735420943280

22. Huang CY, Wu MY, Wang HC, Liao YC, Tou SI, Yen HR. Chinese herbal medicine decreases incidence of cirrhosis in patients with non-alcoholic fatty liver disease in Taiwan: a propensity-score matched cohort Study. J Altern Complement Med. 2021. doi:10.1089/acm.2020.0494

23. Hsing AW, Ioannidis JP. Nationwide Population Science: lessons From the Taiwan National Health Insurance Research Database. JAMA Intern Med. 2015;175(9):1527-1529. doi:10.1001/ jamainternmed.2015.3540.

24. Choong MY, Tou SI, Chang CL, Kuo YH, Huang CY. Association of availability of tap water system with admission rate after 30 days among burn injury patients: a nationwide population-based study. Burns. 2020;46(8):1813-1819. doi:10. 1016/j.burns.2020.06.017
25. Austin PC. Balance diagnostics for comparing the distribution of baseline covariates between treatment groups in propensity-score matched samples. Stat Med. 2009;28(25):3083-3107. doi:10.1002/ sim.3697.

26. Yao XI, Wang X, Speicher PJ, et al. Reporting and guidelines in propensity score analysis: a systematic Review of cancer and cancer surgical studies. J Natl Cancer Inst. 2017;109(8):djw323. doi:10.10 93/jnci/djw323.

27. Chen LY, Yen HR, Sun MF, Lin CL, Chiang JH, Lee YC. Acupuncture treatment is associated with a decreased risk of developing stroke in patients with depression: a propensity-score matched cohort study. J Affect Disord. 2019;250:298-306. doi:10.1016/j. jad.2019.03.020.

28. Goodwin GM, Emsley R, Rembry S, Rouillon F; Agomelatine Study Group. Agomelatine prevents relapse in patients with major depressive disorder without evidence of a discontinuation syndrome: a 24-week randomized, double-blind, placebo-controlled trial. J Clin Psychiatry. 2009;70(8):1128-1137. doi:10.4088/JCP.08m04548

29. Lau B, Cole SR, Gange SJ. Competing risk regression models for epidemiologic data. Am J Epidemiol. 2009;170(2):244-256. doi:10. 1093/aje/kwp107.

30. Ho FM, Huang PJ, Lo HM, et al. Effect of acupuncture at nei-kuan on left ventricular function in patients with coronary artery disease. Am J Chin Med. 1999;27(2):149-156. doi:10.1142/S0192415X99 000197.

31. Zhao L, Li D, Zheng H, et al. Acupuncture as adjunctive therapy for chronic stable angina: a randomized clinical Trial. JAMA Intern Med. 2019;179(10):1388-1397. doi:10.1001/jamainternmed.2019.2407.

32. Shimizu Y, Minatoguchi S, Hashimoto K, et al. The role of serotonin in ischemic cellular damage and the infarct size-reducing effect of sarpogrelate, a 5-hydroxytryptamine-2 receptor blocker, in rabbit hearts. J Am Coll Cardiol. 2002;40(7):1347-1355. doi:10.1016/s07 35-1097(02)02158-7.

33. Ashton JH, Benedict CR, Fitzgerald C, et al. Serotonin as a mediator of cyclic flow variations in stenosed canine coronary arteries. Circulation. 1986;73(3):572-578. doi:10.1161/01.cir.73.3.572

34. Musselman DL, Evans DL, Nemeroff CB. The relationship of depression to cardiovascular disease: epidemiology, biology, and treatment. Arch Gen Psychiatry. 1998;55(7):580-592. doi:10.1001/archpsyc.55. 7.580

35. Vikenes K, Farstad M, Nordrehaug JE. Serotonin is associated with coronary artery disease and cardiac events. Circulation. 1999;100 (5):483-489. doi:10.1161/01.cir.100.5.483

36. Zhang ZJ, Ng R, Man SC, et al. Use of electroacupuncture to accelerate the antidepressant action of selective serotonin reuptake inhibitors: a single-blind, randomised, controlled study. Hong Kong Med J. 2013;19(Suppl 9):12-16.

37. Le JJ, Yi T, Qi L, et al. Electroacupuncture regulate hypothalamic-pituitary- adrenal axis and enhance hippocampal serotonin system in a rat model of depression. Neurosci Lett. 2016;615:66-71. doi:10.1016/j.neulet.2016.01.004

38. Lee BH, Kim YK. Increased plasma VEGF levels in major depressive or manic episodes in patients with mood disorders. J Affect Disord. 2012;136(1-2):181-184. doi:10.1016/j.jad.2011.07.021

39. Painovich J, Phancao A, Mehta P, et al. A randomized, controlled pilot study of the effects of acupuncture on circulating endothelial progenitor cells in coronary heart disease. Integr Med. 2014;13 (2):27-33.

40. King CR, Knutson KL, Rathouz PJ, et al. Short sleep duration and incident coronary artery calcification. JAMA. 2008;300(24):28 59-2866. doi:10.1001/jama.2008.867

41. Mooe T, Franklin KA, Holmström K, Rabben T, Wiklund U. Sleepdisordered breathing and coronary artery disease: long-term prognosis. Am J Respir Crit Care Med. 2001;164(10 Pt 1):19 10-1913. doi:10.1164/ajrccm.164.10.2101072 
42. Chung KF, Yeung WF, Yu BYM, Zhang SP, Zhang ZJ. Combined electroacupuncture and auricular acupuncture for primary insomnia: a randomised controlled trial of dose-response effect. Hong Kong Med J. 2019;25 Suppl 2(1):28-33.

43. Spence DW, Kayumov L, Chen A, et al. Acupuncture increases nocturnal melatonin secretion and reduces insomnia and anxiety: a preliminary report. J Neuropsychiatry Clin Neurosci. 2004;16 (1):19-28. doi:10.1176/jnp.16.1.19

44. Calvillo L, Gironacci MM, Crotti L, Meroni PL, Parati G. Neuroimmune crosstalk in the pathophysiology of hypertension. Nat Rev Cardiol. 2019;16(8):476-490. doi:10.1038/s41569-019-0178-1

45. Petrie JR, Guzik TJ, Touyz RM. Diabetes, hypertension, and cardiovascular disease: clinical insights and vascular mechanisms. Can J Cardiol. 2018;34(5):575-584. doi:10.1016/j.cjca.2017.12.005

46. Wang Y, Zhang Y, Wang W, Cao Y, Han JS. Effects of synchronous or asynchronous electroacupuncture stimulation with low versus high frequency on spinal opioid release and tail flick nociception. Exp Neurol. 2005;192(1):156-162. doi:10.1016/j.expneurol.2004.11.003

47. Torres-Rosas R, Yehia G, Peña G, et al. Dopamine mediates vagal modulation of the immune system by electroacupuncture. Nat Med. 2014;20(3):291-295. doi:10.1038/nm.3479
48. Chen YH, Lee HJ, Lee MT, et al. Median nerve stimulation induces analgesia via orexin-initiated endocannabinoid disinhibition in the periaqueductal gray. Proc Natl Acad Sci U S A. 2018;115(45): E10720-E10729. doi:10.1073/pnas.1807991115

49. Zijlstra FJ, van den Berg-de Lange I, Huygen FJ, Klein J. Antiinflammatory actions of acupuncture. Mediators Inflamm. 2003;12 (2):59-69. doi:10.1080/0962935031000114943

50. Ballegaard S, Jensen G, Pedersen F, Nissen VH. Acupuncture in severe, stable angina pectoris: a randomized trial. Acta Med Scand. 1986;220(4):307-313. doi:10.1111/j.0954-6820.1986.tb02770.x

51. Gao J, Fu W, Jin Z, Yu X. Acupuncture pretreatment protects heart from injury in rats with myocardial ischemia and reperfusion via inhibition of the beta(1)- adrenoceptor signaling pathway. Life Sci. 2007;80(16):1484-1489. doi:10.1016/j.1fs.2007.01.019

52. Huang CY, Kuo YH, Chuang ST, Yen HR, Tou SI. The experience of executing preventive measures to protect a nursing home in Taiwan from a COVID-19 outbreak. Eur Geriatr Med. 2021;1-9. doi:10. 1007/s41999-021-00459-7
Neuropsychiatric Disease and Treatment

\section{Publish your work in this journal}

Neuropsychiatric Disease and Treatment is an international, peerreviewed journal of clinical therapeutics and pharmacology focusing on concise rapid reporting of clinical or pre-clinical studies on a range of neuropsychiatric and neurological disorders. This journal is indexed on PubMed Central, the 'PsycINFO' database and CAS, and
Dovepress

is the official journal of The International Neuropsychiatric Association (INA). The manuscript management system is completely online and includes a very quick and fair peer-review system, which is all easy to use. Visit http://www.dovepress.com/testimonials.php to read real quotes from published authors. 